\title{
A spatially resolved optical emission sensor for plasma etch monitoring
}

\author{
Steven Shannon, James Paul Holloway, Kirk Flippo, and M. L. Brake \\ Department of Nuclear Engineering and Radiological Sciences, University of Michigan, Ann Arbor, \\ Michigan 48109
}

(Received 13 June 1997; accepted for publication 14 July 1997)

\begin{abstract}
A spatially resolved optical emission spectroscopy sensor has been developed, and the resulting reconstructed radial emission profiles from an ArI and ArII line compare well with Ar sputter etch uniformity profiles. The new sensor collects light from a wedge shaped field of view, and is rotated around a single collection point in order to observe the entire plasma through a relatively small viewpoint. (C) 1997 American Institute of Physics. [S0003-6951(97)02937-9]
\end{abstract}

A correlation between glow discharge emission profiles and reactive ion etch depth profiles has been noted by a few authors. ${ }^{1-5}$ Exploitation of this fact could provide an optical sensor that is capable of quickly predicting the etch uniformity profile in a plasma processing tool, and could allow both efficient process uniformity optimization (without the need to examine etch profiles) and real-time process monitoring. In order to achieve these aims, however, practical sensor designs and emission reconstruction algorithms are needed that can deal with the limited optical access likely in industrial processing tools. In this letter, we will briefly describe a sensor design and reconstruction technique that have been developed with this process monitoring goal in mind.

We have simultaneously performed an Ar sputter etch of $\mathrm{SiO}_{2}$ from 4 in. Si wafers and measured plasma emission of the ArI $(750.4 \mathrm{~nm})$ and ArII $(487.9 \mathrm{~nm})$ spectral lines using our optical sensor. These experiments were conducted at a range of setpoints, varying pressure (25-125 mT), Ar flow rate $(10-25 \mathrm{sccm})$, and power $(75-175 \mathrm{~W})$. In all cases, the reconstructed argon emission profiles were found to agree well with the measured sputter etch depth.

The sensor itself is an optical train consisting of a rectangular entrance aperture $(1.6 \times 6.4 \mathrm{~mm}$ in size $)$ followed by a cylindrical lens, with a $200 \mu \mathrm{m}$ slit placed at its focal length. This slit is immediately followed by a fiber optic cable, which by virtue of the preceding optics, is collecting light from a wedge shaped field of view. The sensor is mounted on a rotating stage near a 6 in. diam viewport on the University of Michigan GEC reference cell. ${ }^{6}$ The fiber optic from the sensor is coupled to an Acton Research AM510 monochrometer, and a photomultiplier tube (PMT) is placed at the output slit. The PMT voltage signal provides the raw data for our measurements. The sensor is swept across the plasma in $0.2^{\circ}$ increments, and the PMT voltage measured as a function of angle. A schematic of the experimental setup, including the sensor, is shown in Fig. 1.

The vertical acceptance angle of the sensor is $0.078^{\circ}$. The horizontal acceptance angle of the sensor is about $3^{\circ}$ (from the optic axis), but its response to light is not uniform over this range of angles; photons entering along the optical axis are more efficiently detected than those entering at angles off of the optical axis. This nonuniform response is

a)Electronic mail: brake@umich.edu measured as a function of horizontal angle using a collimated $\mathrm{Kr}$ pen lamp.

In order to reconstruct the plasma emission profile that produces the measured signal, the emission is modeled as a concentric set of 60 uniform rings of emission; this is more than sufficient to resolve all the features of the emission. The determination of the total intensity of each such ring provides the radial profile of the plasma emission. Each such ring gives a characteristic contribution to the measured signal, and the shape of this contribution is known because we have measured the sensor response as a function of angle with the calibration lamp. Effectively, therefore, the measured signal must be approximated by the sum of these characteristic contributions. A straightforward least-squares formulation of this is ill posed (i.e., tiny errors in measured data result in huge errors in reconstructed emission) and, therefore, not used. Instead, we use a generalized Tkhonov regularization ${ }^{7,8}$ with a finite difference first derivative of the emission as a regularizing operator. The regularization parameter is selected using the optimization technique described by Reginska. ${ }^{8}$ This method has proved very robust; the results are independent of the number of rings used and can tolerate all but the most severe experimental noise in our measurements.

Figure 2 shows a comparison between the measured etch depth profiles (data points) along an unmasked $\mathrm{SiO}_{2}$ coated $\mathrm{Si}$ wafer and the reconstructed 750.4 and $487.9 \mathrm{~nm}$ plasma emission profiles (solid and dashed line, respectively) for two different sets of operating parameters; the etch depth

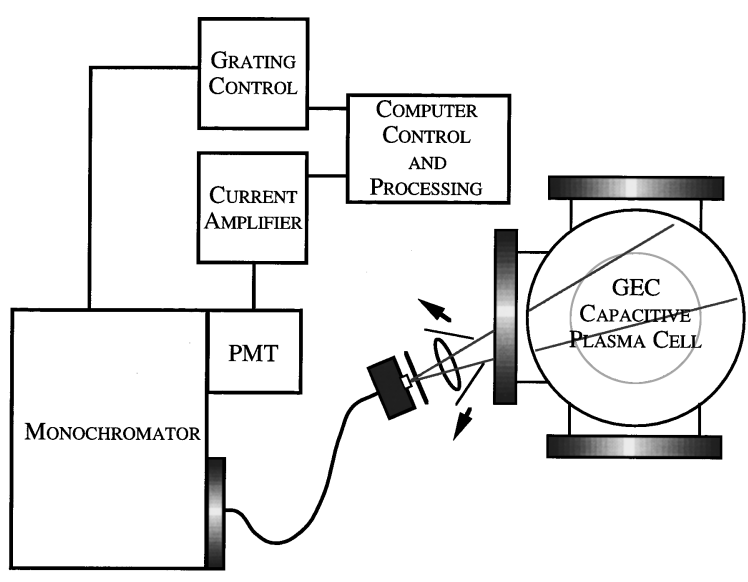

FIG. 1. A schematic diagram of the sensor and experiment. 

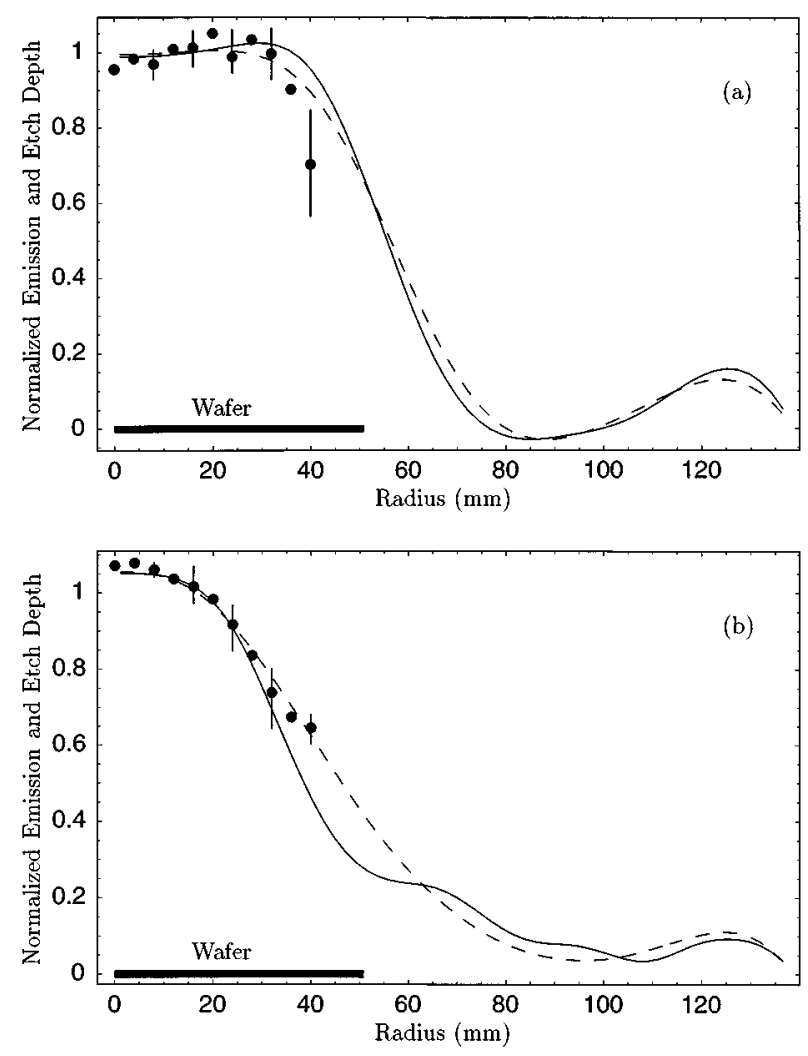

FIG. 2. Two examples of the reconstructed emission profiles (solid line: $750.4 \mathrm{~nm}$; dashed line: $487.9 \mathrm{~nm}$ ) superimposed on etch depth uniformity data (points). Both cases are for $175 \mathrm{~W}$ input rf power and $35 \mathrm{sccm}$ Ar flow rate, but for two different pressures: $125 \mathrm{mT}$ (a) and $25 \mathrm{mT}$ (b). The peak etch depths are $340 \AA$ (a) and $740 \AA$ (b).

was measured using a spectral reflectometer. The etch depth and emission profiles are normalized to have an average value of 1 over the inner $28 \mathrm{~mm}$ of the radius. There is a good quantitative agreement between the shape of the measured etch depth profiles and the reconstructed emission profiles at other pressures, powers, and flow rates as well.
The reconstructed emission shown in Fig. 2 probably falls off too slowly at the electrode edge (colocated with the wafer edge). This is expected in part because the regularization operator (first derivative) prevents the reconstructed emission from changing too rapidly with the radius. An improved regularization operator is needed to allow the rapid quenching of the reconstructed plasma emission at the electrode edge. The second effect that blurs the true plasma edge is the reflection of the plasma emission from the interior of the vacuum chamber. This blurring of the emission is far less pronounced near the center of the plasma because emission from large radii has very little effect on the shape of the signal at small angles, toward the center of the discharge where the wafer is located.

The sensor system and reconstruction algorithm that we have briefly described in this letter is able to measure and reconstruct plasma emission profiles that correspond very well with etch depth uniformity measurements. Our conclusion is that this sensor system provides a reliable optical method to predict plasma Ar sputter etch uniformity of $\mathrm{SiO}_{2}$ coated Si wafers.

This research was supported by SEMATECH Contract No. Etch001, NSF Grant No. ECS-9359344, and SRC Contract No. 96-FC-085.

${ }^{1}$ H. M. Anderson and M. P. Splichal, Proc. SPIE 2091, 333 (1994).

${ }^{2}$ M. Buie, J. Pender, J. Soniker, M. Elta, and M. Brake, J. Vac. Sci. Technol. A 13, 1930 (1995).

${ }^{3}$ M. Brake, J. Pender, M. Buie, A. Ricci, J. Soniker, P. Pochan, and P. Miller, J. Res. Natl. Inst. Stand. Technol. 100, 441 (1995).

${ }^{4}$ M. J. Buie, J. T. P. Pender, J. P. Holloway, T. Vincent, P. Ventzek, and M. Brake, J. Quant. Radiat. Spectrosc. 55, 231 (1995).

${ }^{5}$ M. Buie, J. Pender, J. Holloway, and M. Brake, IEEE Trans. Plasma Sci. 24, 111 (1996).

${ }^{6} \mathrm{P}$. Hargis et al., Rev. Sci. Instrum. 65, 140 (1994).

${ }^{7}$ V. B. Glasko, Inverse Problems of Mathematical Physics (AIP, New York, 1988).

${ }^{8}$ Teresa Reginska, SIAM J. Sci. Comput. 17, 740 (1996). 